\title{
Optimization of Cooperative Spectrum Sensing with Energy Detection in Cognitive Radio Networks using Voting Rule
}

\author{
Tina Sain \\ Research Scholar \\ Baba Farid College of Engineering and technology, \\ Bathinda, India
}

\author{
Kartik Sharma \\ Assistant Professor \\ Baba Farid College of Engineering and technology, \\ Bathinda, India
}

\begin{abstract}
We consider cooperative spectrum sensing in which multiple cognitive radios collaboratively detect the spectrum holes through energy detection and investigate the optimality of cooperative spectrum sensing with an aim to optimize the detection performance in an efficient and implementable way. The optimal voting rule has been derived for any detector applied to cooperative spectrum sensing. Also, detection threshold is optimized when energy detection is employed. Finally, a fast spectrum sensing algorithm for a large network is proposed which requires fewer than the total number of cognitive radios in cooperative spectrum sensing while satisfying a given error bound.
\end{abstract}

\section{Keywords}

Spectrum Sensing, Cognitive Radio Network, Voting Rule, Thresholding, Energy Optimization.

\section{INTRODUCTION}

Cognitive Radio (CR) has been carried out as a promising technology to enhance the radio spectrum utilization. The main functions of $\mathrm{CR}$ are: [1] spectrum sensing: detecting unused spectrum, [2] spectrum management: capturing the best available spectrum to satisfy the user communication demand, spectrum mobility: maintaining seamless communication, and spectrum sharing: providing the fair spectrum access among coexisting users. Cognitive radio technique has been recently proposed as a novel approach for improving the utilization of the precious limited radio resources, by allowing secondary users access primary users' spectrum if not used. There are two major confronts for cognitive radio: spectrum sensing and hidden terminal problem [3]. In this paper, a novel network structure is proposed to separate the spectrum sensing task from the secondary users. The fusion center for the secondary users needs to place fusion center within the primary users' networks. These fusion centers thus, sense the primary users' activity. The fusion center also decides whether to admit a secondary user's transmission [4].

Other great challenges of implementing spectrum sensing is the hidden terminal problem, Due to hidden terminal problem, a cognitive radio may fail to notice the presence of the PU and then will access the licensed channel and cause interference to the licensed system. Moreover, for a better performance in spectrum sensing, the local sensing results are sent to central entity, called Fusion Center (FC) [5]. One of the great challenges of implementing spectrum sensing is the hidden terminal problem, which arises when the cognitive radio is shadowed, in severe multipath fading or inside buildings with high penetration loss, while a primary user (PU) is operating in the vicinity. So in this paper hidden terminal problem will be solved using voting rule.

\subsection{Hidden Terminal Problem}

Hidden terminal problem occurs when a node is visible from the access point but cannot communicate to the nodes within communication distance. Star network is the best example. One of the well-known hidden node problem in wireless networks is a three node problem [6]. The hidden terminal problem can be solved by fusion center with cognitive radio network. For example, the node A try to broadcast to node B and node $\mathrm{C}$ try to broadcasts to node $\mathrm{B}$ during the same time. The node $\mathrm{A}$ and node $\mathrm{C}$ cannot see each other and trying to broadcast at the same time. During the process the packets collide at node $\mathrm{B}$. we have reduce this hidden terminal problem by using threshold algorithm and voting algorithm. Both these algorithm reduce the total error rate in cognitive radio network. When hidden terminal problem reduce then total error rate are also reduce and throughput will be increases automatically in the cognitive radio network [7]. It should be mentioned that optimal spectrum sensing under data fusion was investigated in, where the optimal linear function of weighted data fusion has been obtained. In other recent works [8], [9], optimal sensing through tradeoff was studied. Also, Optimal distributed signal detection with likelihood ratio test using reporting channels from the CRs to the fusion center has been dealt with in [10].

\section{SPECTRUM SENSING}

We consider a CR network composed of CRs (secondary users) and a common receiver, as shown in Fig. We assume that each CR performs spectrum sensing autonomous and then the local decisions are sent to the common receiver which can fuse all available decision information to infer the absence or presence of the PU. The essence of spectrum sensing is a Binary hypothesis-testing problem:

Ho: primary user is absent

$\mathrm{H}_{1}$ : primary user is in operation.

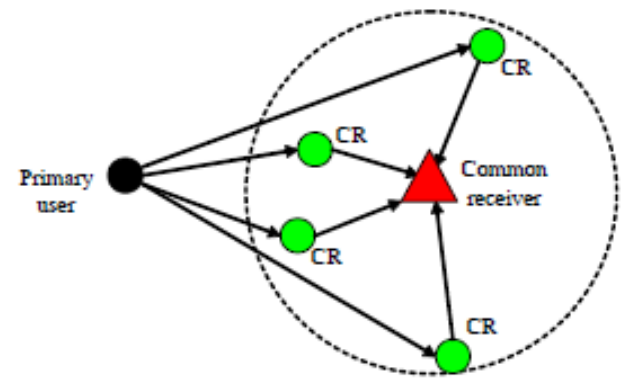

Fig. 1: Spectrum sensing structure in a cognitive radio network. 


\section{MOTIVATION}

One of the major challenges of cognitive radio hidden terminal problem is which occurs when the cognitive radio is shadowed in severe multipath fading or inside buildings with high penetration loss, while a primary user (PU) is operating in the neighbor [11]. Due to the hidden terminal problem, a cognitive radio may fall short to notice the presence of the PU and then will access the licensed channel and cause interference to the licensed system. It has been shown that spectrum sensing performance can be greatly improved with an increase of the number of cooperative partners. In this work, we consider the optimization of cooperative spectrum sensing with energy detection to minimize the total error rate [12].

\section{WORKING METHODOLOGY}

We consider a CR network composed of CRs (secondary users) and a common receiver, as illustrated in Fig.2. We assume that each CR performs spectrum sensing independently and then the local decisions are sent to the common receiver which can fuse all available decision information to infer the absence or presence of the PU. The spirit of spectrum sensing is a binary hypothesis-testing problem:

Ho: Primary user is absent

$\mathrm{H}_{1}$ : Primary user is in operation.

We consider a CR network composed of CRs (secondary users) and a common receiver (Fusion Center), we assume that each CR performs spectrum sensing independently and then the local decisions are sent to the common receiver which can fuse all available decision information to infer the absence or presence of the PU.

To the Optimal rule, we have to find out the number of $\mathrm{Ks}$ (Value of voting rule), The results here shows the total error rate in terms of the detection threshold for various voting rules from $n=1$ to $n=10$ in a cognitive network with 10 users.

It can be observed that the optimal voting rule over all the examined range of detection thresholds is $n=5$. However, for a fixed very small threshold. The all nodes in CRN first, communicate to the common receiver(fusion center) under this voting rule, each of the node with the value of its $\mathrm{K}$ reach to the fusion center and update the fusion center regarding its current resources and threshold value.

Once the communication get started these nodes start transferring data from one another and also update the fusion center so that the optimal path can be obtained for the routing with respect to TH value.

The graphs showing the value of TER vs TH value represents the state of the CRN which can be taken as throughput maximization in current CRN.

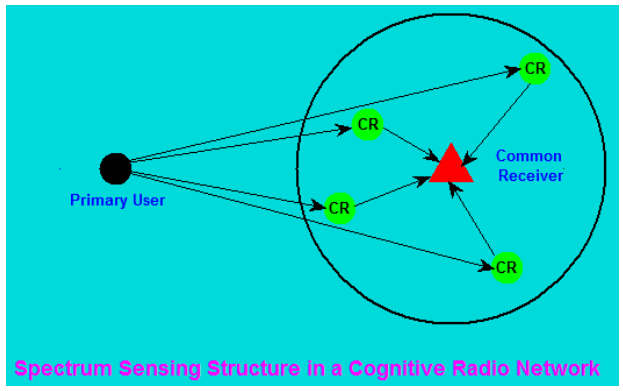

Fig.2: Spectrum Sensing Structure
In the following we only consider the spectrum sensing at CR i. The sensing method is to decide between the following two hypotheses:

$\begin{array}{lc}X_{i}(t)=w_{i}(t), & H_{o}, \\ X_{i}(t)=h_{i}(t) s(t)+w_{i}(t), & H_{1},\end{array}$

Where $x_{i}(t)$ is the received signal at the ith CR in time slot $t$, $\mathrm{s}(\mathrm{t})$ is the $\mathrm{PU}$ signal, $\mathrm{w}_{\mathrm{i}}(\mathrm{t})$ is the additive white Gaussian noise (AWGN), and $h_{i}(t)$ denotes the complex channel gain of the sensing channel between the PU and the ith CR. We assume that the sensing time is smaller than the coherence time of the channel. Then, the sensing channel $\mathrm{h}(\mathrm{t})$ can be viewed as timeinvariant during the sensing process. Without loss of generality, we denote $h(t)$ as $h_{i}$

\subsection{Optimization}

In this section, we consider the optimality of cooperative spectrum sensing when energy detection and decision fusion are applied.

\section{A. Optimal Voting Rule}

To apply the Optimal rule, we have to find out the number of Ks, So before we delve into pursuing the exact optimal solution of $n$, let us get some intuitive results from figure in experiments chapter, shows the total error rate in terms of the detection threshold for various voting rules from $n=1$ to $n=$ 10 in a cognitive network with 10 users. It can be observed that the optimal voting rule over all the examined range of detection thresholds is $n=5$. However, for a fixed very small threshold, the optimal rule is the AND rule, i.e., $n=10$. Meanwhile, for a fixed very large threshold, the OR rule, i.e., $n=1$, tends to be optimal. Next, we give the exact solution of the optimal $n$ in the following proposition.

\section{B. Optimal Energy Detection Threshold}

As Qf + Qm has been find out above, so Threshold detection will be found out using:

$\gamma=\arg \min (\mathrm{Qf}+\mathrm{Qm})$

\section{Optimal Number of Cognitive Radios}

In a cognitive radio network with a big number of CRs, cooperative spectrum sensing may become impractical because in a time slot only one CR should send its local decision to the common receiver so as to separate decisions at receiver end with ease. Consequently, it may make the whole sensing time intolerably long. This issue can be addressed by allowing the CRs to send the decisions concurrently. But it may complicate the receiver design when separating the decisions from different CRs. Another potential solution is to send the decisions on orthogonal frequency bands, but this requires a large portion of available bandwidth. To address these issues, we propose next an efficient sensing algorithm which relies on the transmission of decision in one time slot for one CR but guarantees a target error bound by requiring a few CRs in cooperative spectrum sensing instead of all of them. To do so, we consider that Suppose that the SNR $\gamma$ and $\lambda$ are known, First we assume that $K(1 \leq K \leq K)$ is the least required number of $\mathrm{CRs}$ required in cooperative spectrum sensing so as to satisfy ( $\mathrm{Qf}+\mathrm{Qm}) \leq \epsilon$. Then, from Proposition 1, w can see that the optimal voting rule for cooperative spectrum. 


\section{SIMULATION MODEL}

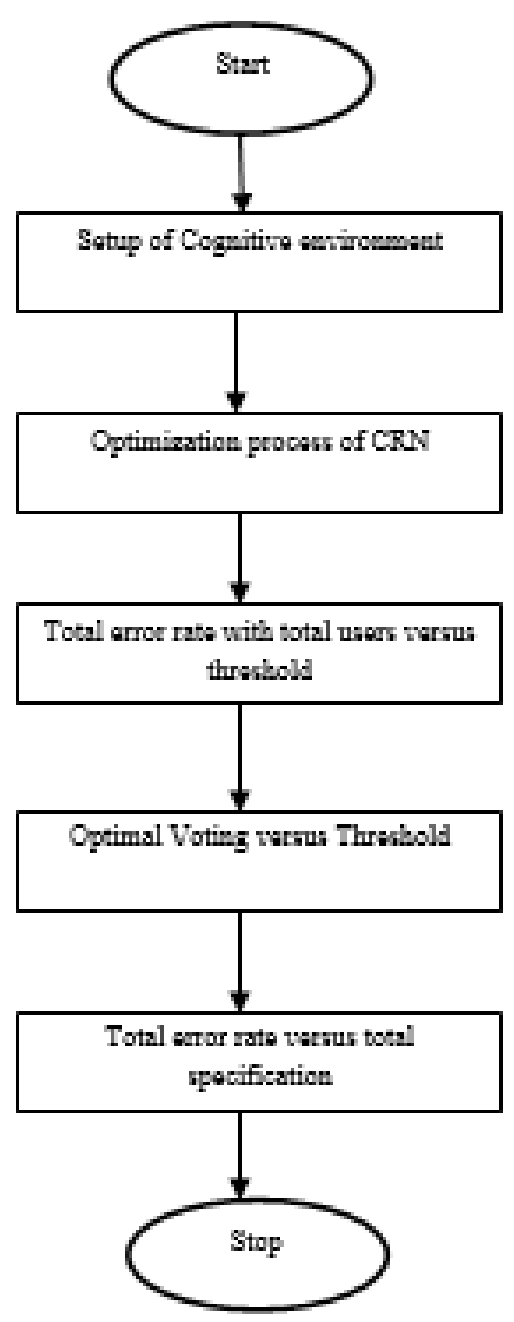

Fig.3: Proposed Flowchart

\section{RESULTS AND IMPLEMENTTAIONS}

The whole simulation has been taken place in MATLAB 7.10 using voting rule to solve the hidden terminal problem.

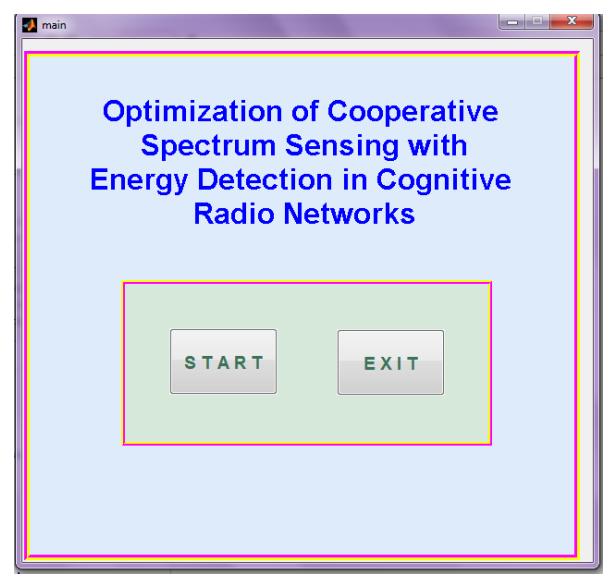

Fig.4: Start-up page

The above figure is the start-up page of our CR network In order to proceed in the CR network, we can click START button otherwise, and EXIT button can be selected.

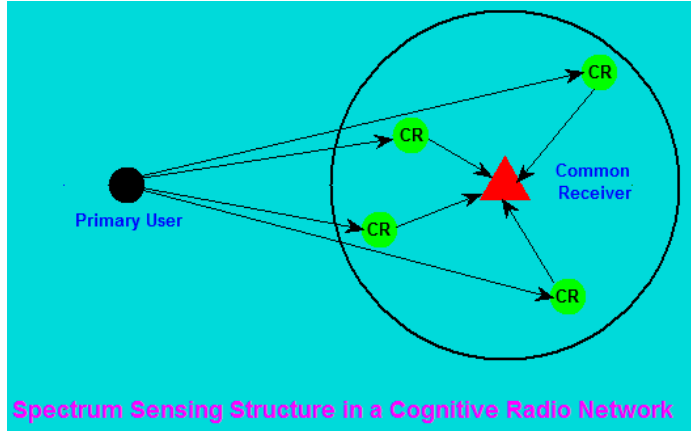

Fig.5: Spectrum sensing structure

In the above figure, we consider a CR network composed of K CRs (secondary users) and a similar receiver. We suppose that each CR performs spectrum sensing independently and then the local decisions are sent to the common receiver which can fuse all available decision information to infer the absence or presence of the PU.

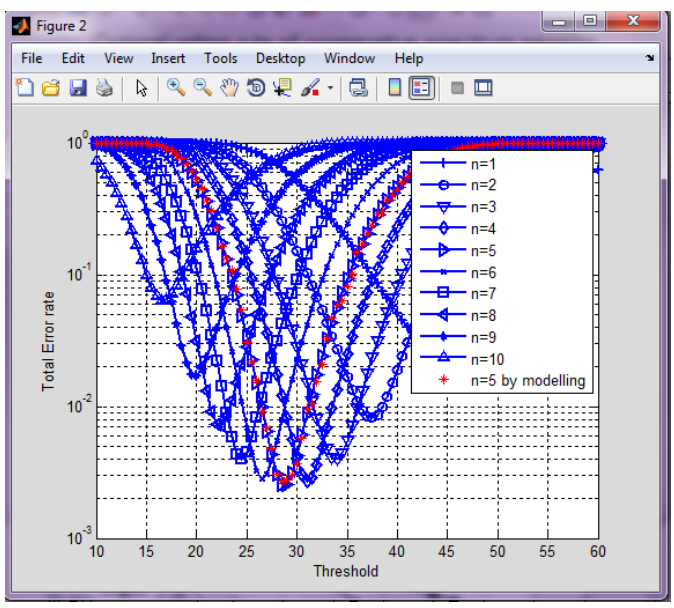

Fig.6: Total error rate versus threshold

Above Figure shows, total error rate in terms of the detection threshold for various voting rules from $n=1$ and $n=10$, in a cognitive network with 10 users. It can be observed from Fig. That the optimal voting rule over all the examined range of detection thresholds is $\mathrm{n}=5$ However, for a fixed very small threshold, the optimal rule is the AND rule, i.e., $\mathrm{n}=10$. Meanwhile, for a fixed very large threshold, the OR rule, i.e. $\mathrm{n}$ $=10$ tends to be optimal.

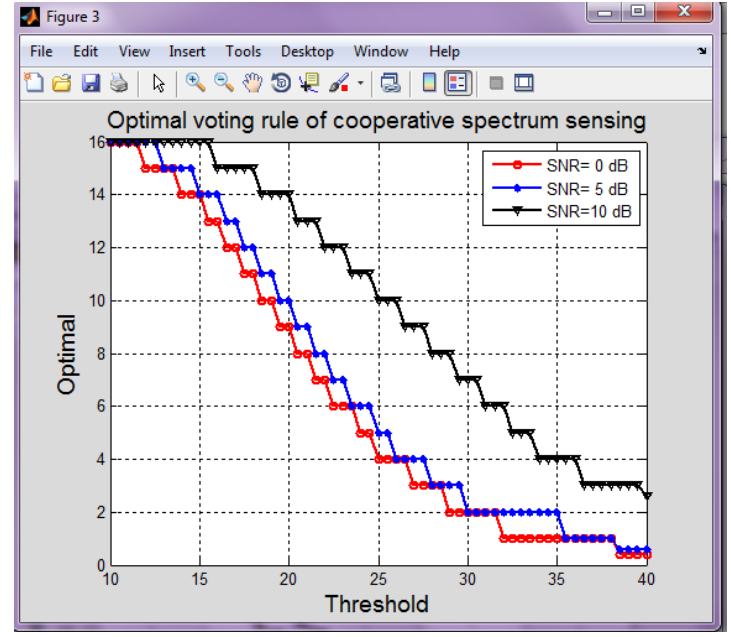

Fig.7: Optimal versus threshold 
The above Figure shows the exact solution of $\mathrm{n}$ in terms of detection threshold evaluated from $\mathrm{n}=\min (\mathrm{K},[\mathrm{K} / 1+\mathrm{a}])$. The results validate the remarks that we have made below: Usually, $\mathrm{Pf}$ and $\mathrm{Pm}$ have the same order, i.e., $\mathrm{a} \approx 1$. Thus, the most favorable choice of $n=K / 2$. The OR rule is optimal when $\mathrm{a}=\mathrm{K}-1$ This means that $\mathrm{Pf}=\mathrm{Pk}-1$ This implies that $\mathrm{Pf}$ $\ll P$ m for a large value $\mathrm{K}$. This can be achieved when the detection threshold $\lambda$ is very large. The AND rule is optimal when $\mathrm{a}=0$. This is achieved when $\mathrm{Pm} \ll$ i.e., for a very small $\lambda$.

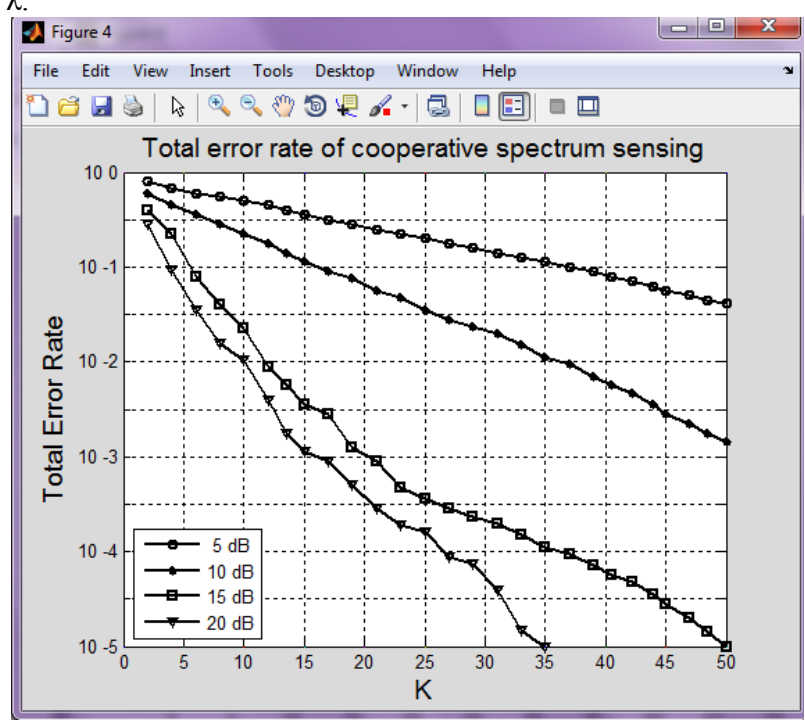

Fig.8: Total error rate versus $\mathrm{K}$

In the above Figure, we find that the smallest numbers of CRs to get the error rate target are 12, 17, and 32 for SNR values of 20,15, 10 and $5 \mathrm{~dB}$, respectively. Thus it specifies that to obtain a required quality-of service it is sufficient to employ minimal cooperation.

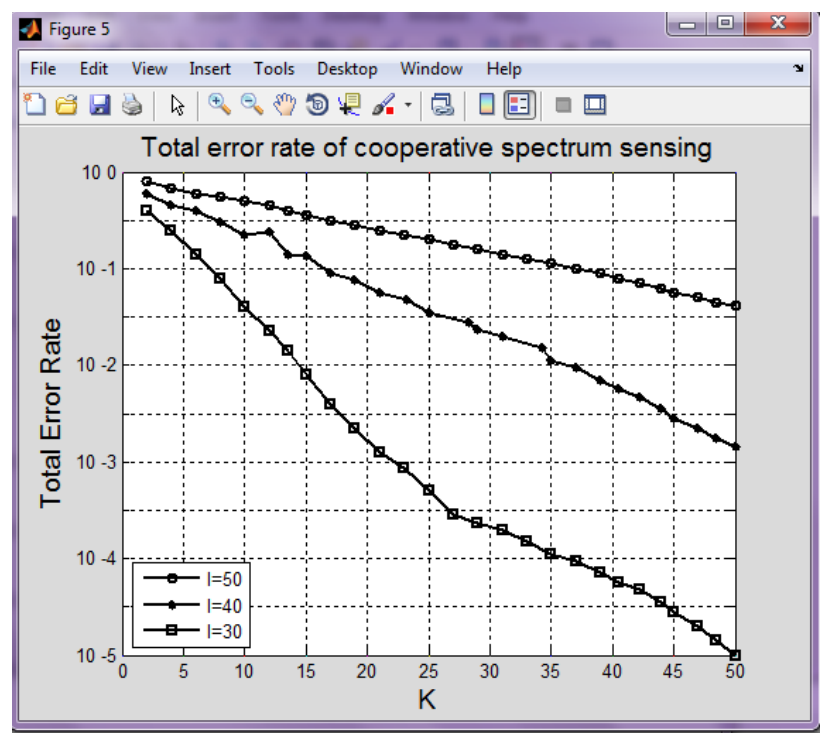

Fig.9: Total error rate versus $K$ from I 50 to 30

The above Figure shows another example of using a few CRs to get a total error rate of 0.01 for energy detection at an SNR of $10 \mathrm{~dB}$ in an AWGN channel. In particular, when the threshold is set as 30 , it is sufficient to consider cooperative spectrum sensing with 8 CRs only for the given target of Qf + $\mathrm{Qm}<.01$.

\section{CONCLUSION AND FUTURE SCOPE}

In a cognitive radio network with such a large number of CRs, it becomes impossible to have cooperative spectrum sensing because in a time slot only one CR should send its local decision to the common receiver so as to separate decisions easily at the receiver end. Hence, the whole sensing time may become intolerably long. To overcome these issues, we propose next an efficient sensing algorithm which relies on the transmission of decision in one time slot for one CR but guarantees a target error bound by requiring a few CRs in cooperative spectrum sensing rather than all of them. Also, we have studied the performance of cooperative spectrum sensing with energy detection in cognitive radio networks. It has been initiated that the optimal decision voting rule to minimize the total error probability is the half-voting rule. Also, a method of numerically getting the optimal detection threshold has been presented. In count, an efficient spectrum sensing algorithm has been proposed which requires fewer than the total number of cognitive radios in cooperative spectrum sensing while satisfying a given error bound.

\section{REFERENCES}

[1] Ian F. Akyildiz, Won-Yeol Lee, Mehmet C. Vuran, Shantidev Mohanty, "NeXt generation/dynamic spectrum access/cognitive radio wireless networks: A survey," Elsevier Computer Network Journal, vol. 50, no. 13, pp. 2127-2159, 2006.

[2] Amir Ghasemi, Communications Research Centre Canada and University of Toronto Elvino S. Sousa, University of Toronto, "Spectrum Sensing in Cognitive Radio Networks: Requirements, Challenges and Design Trade-offs," IEEE Communications Magazine, vol. 46, no. 4, pp. 32-39, April 2008.

[3] Zhu Han and +Hai Jiang, "Replacement of Spectrum Sensing and Avoidance of Hidden Terminal for Cognitive Radio," in IEEE Wireless Communications and Networking Conference, april 2008, pp. 1448-1452.

[4] W. Zhang and K. B. Letaief, "Cooperative spectrum sensing with transmit and relay diversity in cognitive radio networks," IEEE Trans. Wireless Commun., vol. 7 , pp. 4761-4766, Dec. 2008

[5] G. Ganesan and Y. G. Li, "Cooperative spectrum sensing in cognitive radio networks," in Proc. IEEE Symp. New Frontiers Dynamic Spectrum Access Networks (DySPAN'05), Baltimore, USA, Nov. 2005, pp. 137143.

[6] Yenumula B. Reddy, "Cognitive Networks: Detecting Primary signal by Minimizing Hidden Terminal Problem," Science Academy Transactions on Computer and Communication Network (SATCCN)Vol. 2, No. 2, pp. 127-133,June 2012,

[7] Wei Zhang, Member, Ranjan K. Mallik, Senior Member, , and Khaled Ben Letaief, Fellow," Optimization of Cooperative Spectrum Sensing with Energy Detection in Cognitive Radio Networks," IEEE TRANSACTIONS ON WIRELESS COMMUNICATIONS, VOL. 8, NO. 12, DECEMBER 20095761 
[8] Z. Quan, S. Cui, and A. H. Sayed, "Optimal linear cooperation for spectrum sensing in cognitive radio networks," IEEE J. Sel. Topics Signal Process., vol. 2, no. 1, pp. 28-40, Feb. 2008.

[9] E. Peh and Y.-C. Liang, "Optimization for cooperative sensing in cognitive radio networks," in Proc. IEEE Int. Wireless Commun. Networking Conf., Hong Kong, Mar. 2007, pp. 27-32.

[10] Y.-C. Liang, Y. Zeng, E. Peh, and A. T. Hoang, "Sensing-throughput tradeoff for cognitive radio networks," IEEE Trans. Wireless Commun., vol. 7, pp. 1326-1337, Apr. 2008.
[11] S. M. Mishra, A. Sahai, and R. Brodersen, "Cooperative sensing among cognitive radios," in Conf. Rec. IEEE Int. Conf. Commun. (ICC’06), Turkey, June 2006, vol. 4, pp. $1658-1663$.

[12] K. B. Letaief and W. Zhang, "Cooperative communications for cognitive radio," Proc. IEEE, vol. 97, no. 5, pp. 878-893, May 2009.

[13] A. Ghasemi and E. S. Sousa, "Collaborative spectrum sensing for opportunistic access in fading environments," in Proc. IEEE Symp. New Frontiers in Dynamic Spectrum Access Networks (DySPAN'05), Baltimore, USA, Nov. 2005, pp. 131-136. 